Check for updates

Cite this: Phys. Chem. Chem. Phys., 2021, 23, 24699

\title{
First principles simulations of microscopic mechanisms responsible for the drastic reduction of electrical deactivation defects in Se hyperdoped silicon
}

\begin{abstract}
Alberto Debernardi (D)
By first principles simulations we systematically investigate Se hyperdoped silicon by computing, for different types of Se complexes, the formation energy as a function of dopant concentration. We identify the microscopic mechanisms responsible for the dramatic reduction of electrical deactivation defects as the dopant concentration approaches the critical value, $x_{c}$, at which the insulator-to-metal transition occurs. We discuss the electrical properties of Se point defects and Se complexes, shedding light on the formation and the nature of the impurity band in the bandgap and how the presence of different types of complexes may increase the broadening of the impurity band and affects the insulator-to-metal transition. We identify the best doping range in which the properties of the impurity band can be engineered according to the needs of the electronic industry. Simulations of the structural properties of the complexes complete the work. Our findings are relevant for intermediate impurity band applications.
\end{abstract}

Received 26th June 2021

Accepted 8th October 2021

DOI: $10.1039 / \mathrm{d} 1 \mathrm{cp} 02899 \mathrm{e}$

rsc.li/pccp

\section{Introduction}

Silicon hyperdoped (i.e., doped beyond the solid solubility limit) with donor impurities is attracting increasing attention from the electronic industry and materials science community because it is a promising candidate as a building block for the conception of new electronic devices ranging from infrared absorbers, ${ }^{1-3}$ to intermediate band photovoltaics ${ }^{4,5}$ to ultrascaled nano-electronics ${ }^{6}$. The latter application is motivated by the continuous scaling down of Si-based transistors in onchip complementary metal oxide semiconductor (CMOS) devices, which are approaching the $2 \mathrm{~nm}$ technology node. Therefore, the next generation of ultra-scaled CMOS devices requires extremely high free-electron densities, of the order of $10^{21} \mathrm{~cm}^{-3}$ (i.e. one electron per $\mathrm{nm}^{3}$ ) to ensure sufficient free carriers in a transistor to work. ${ }^{7,8}$

Shallow donors, such as group V elements (P, As, or $\mathrm{Sb})$, are traditionally employed as dopants in Si for their low ionization energy, low diffusivity and suitable solid solubility. Unfortunately, their free-electron concentration saturates at around $5 \times 10^{20} \mathrm{~cm}^{-3}:{ }^{9,10}$ beyond this concentration the introduction of further donors does not generate free carriers, thus providing an upper limit for the electron density, preventing the realization of nanometer-size devices.

CNR-IMM, Unit of Agrate Brianza, via C. Olivetti 2, 20864 Agrate Brianza (MB), Italy.E-mail: alberto.debernardi@mdm.imm.cnr.it
In the last few decades, experimental ${ }^{11-13}$ and theoretical ${ }^{14-16}$ works studied the microscopic mechanisms responsible for saturation of free-electron concentration in group $\mathrm{V}$ dopants in Si. The saturation has been attributed to the formation of electrical deactivation defects. According to the literature, these defects have been identified with different types of substitution complexes: the $\mathrm{As}_{n}-\mathrm{V}_{\mathrm{Si}}(n \leq 4)$ model (the clustering around a Si vacancy, $\mathrm{V}_{\mathrm{Si}}$, surrounded by As atoms, ${ }^{12,14-23}$ also invoked to interpret $\mathrm{Sb}_{n}-\mathrm{V}_{\mathrm{Si}}$ clusters ${ }^{24}$ ); the dimers (substitutional dopant in nearest neighbor (NN) lattice sites) ${ }^{25}$ and the defects containing pairs of separated dopant atoms (in next NN lattice sites) without vacancies. ${ }^{25,26}$ These group $\mathrm{V}$ complexes introduce deep acceptor states in the bandgap that deactivate free carriers, thus preventing high electron concentrations. ${ }^{25,26}$

At variance, chalcogen impurities (group VI elements: S, Se, and $\mathrm{Te}$ ) are deep donors in $\mathrm{Si}$, having ionization energy of a few hundreds of $\mathrm{meV}$ at low concentration, while at high concentration they can induce free electrons (in excess of $10^{20} \mathrm{~cm}^{-3}$ ) in $\mathrm{Si}$, accompanied by an insulator-to-metal transition (IMT) ${ }^{27,28}$ Chalcogens show superior electronic properties as Si hyper-dopants than traditional group $\mathrm{V}$ elements: a Te concentration of $1.25 \times 10^{21} \mathrm{~cm}^{-3}$ has been reached in hyperdoped $\mathrm{Si}$ without showing any sign of saturation, ${ }^{6,29}$ while the electron concentration $\left(8.1 \times 10^{20} \mathrm{~cm}^{-36}\right)$ in the same samples nearly scored the target of $10^{21} \mathrm{~cm}^{-3}$ required for the next generation of the Si technology node. ${ }^{7,9,30}$

Furthermore, hyper-doping establishes a new materials playground to investigate impurity mediated IMTs in 
semiconductors, that has been largely studied both for its interest in fundamental physics and for its relevance in technological applications. Previous studies about IMT in $\mathrm{S}^{27,31}$ and $\mathrm{Se}^{28}$ hyperdoped Si have been focused on the single substitutional impurities, while recently, first principles simulations have enlightened the role of dimers as a driving force of IMT in Te hyperdoped $\mathrm{Si}^{6,29}$

Computational studies are therefore desirable to validate this scenario, by considering other chalcogen dopants, and additional types of chalcogen complexes, to provide novel insights into the microscopic mechanisms governing the hyperdoped regime in $\mathrm{Si}$.

In this work we present first principles simulations of the formation energy of different complexes in Se hyperdoped Si in a wide range of dopant concentrations.

This article is structured as follows: in Section II we describe the computational techniques used in the work; in Section III.A we display our results for the formation energy of the different types of defects investigated as a function of dopant concentration; for each defect, we present our first principles results for the electronic, and the structural properties, respectively in Sections III.B and III.C. In Section IV we discuss our finding in relation to applications in which se hyperdoped silicon can be used as building block in innovative devices based on the formation of an intermediate impurity band in the electronic gap. Finally, in Section V we present our conclusions and summarize our results.

\section{Computational methods}

In our simulations we consider Se interstitial $\mathrm{I}_{\mathrm{Se}}$, single Se substitutional, $\mathrm{Se}_{\mathrm{Si}}$, Se dimers $\mathrm{Se}_{\mathrm{Si}}-\mathrm{Se}_{\mathrm{Si}}$ and complexes formed by a $\mathrm{Si}$ vacancy $\mathrm{V}_{\mathrm{Si}}$ surrounded by $m$ Se atoms, denoted as $\left(\mathrm{Se}_{\mathrm{Si}}\right)_{m}-\mathrm{V}_{\mathrm{Si}}, m=1,2,3,4$. Although the latter complexes have been extensively studied for group V donors (mainly As), the possible role of the clustering of chalcogen atoms around a vacancy seems still unexplored by $a b$ initio simulations. Geometrical models of neutral single vacancy-selenium complexes are determined from first principles by plane-wave pseudopotentials techniques. We will present the formation energy of these complexes as a function of the Se concentration, and we will discuss electrical properties to enlighten the advantages of chalcogen atoms as dopants in forthcoming nanometer-size devices based on hyperdoped $\mathrm{Si}$.

Our simulations are obtained using the super-cell method within the single complex model (SCM); accuracy and limit of SCM, and all computational details are discussed in Appendix A.

\section{Results}

\section{A Defect formation energy}

We denote with $D$ a generic defect among the ones considered in the present work (i.e. $D \in \mathrm{I}_{\mathrm{Se}}, \mathrm{Se}_{\mathrm{Si}}, \mathrm{Se}_{\mathrm{Si}_{\mathrm{i}}}-\mathrm{Se}_{\mathrm{Si}}$ and $\left(\mathrm{Se}_{\mathrm{Si}}\right)_{m}-\mathrm{V}_{\mathrm{Si}}$, $m=1,2,3$, and 4$)$. For the defect $D$, the formation energy per $S e$ atom as a function of Se concentration $x$, in hyperdoped silicon $\left(\mathrm{Si}_{1-x} \mathrm{Se}_{x}\right)$, reads

$$
\Delta E_{D}^{\mathrm{Form}}(x) \equiv\left[E_{D}(x)-N_{\mathrm{Si}} \mu_{\mathrm{Si}}-N_{\mathrm{Se}} \mu_{\mathrm{Se}}\right] / N_{\mathrm{Se}}
$$

where $E_{D}(x)$ is the total energy of the supercell with $D$ (a point defect or a complex), $N_{\mathrm{Si}}$ and $N_{\mathrm{Se}}$ are the numbers of Si and Se atoms in the supercell, respectively, and $\mu_{\mathrm{Si}}$ and $\mu_{\mathrm{Se}}$ correspond to the chemical potentials of bulk $\mathrm{Si}$ and bulk $\mathrm{SiSe}_{2}$ at equilibrium with each other.

In Fig. 1 and 2 we display our computed formation energy at different doping concentrations for all complexes investigated. Note that, accordingly to the choice of the chemical potentials, the zero of the energy corresponds to the phase separation of $\mathrm{Si}_{1-x} \mathrm{Se}_{x}$ into bulk $\mathrm{Si}$ and $\mathrm{SiSe}_{2}$, and all Se complexes present positive formation energy, in agreement with the experimental fact that, in the concentration range considered, $\mathrm{Si}$ is doped beyond the Se solid solubility limit. ${ }^{6,32}$

By the comparison of the energy scales of the two figures we can immediately appreciate that at high concentration $(x \gtrsim 0.45$ at\%) the formation energies of the complexes displayed in Fig. $2\left(\mathrm{I}_{\mathrm{Se}}\right.$ and $\left.\mathrm{Se}_{\mathrm{Si}}-\mathrm{V}_{\mathrm{Si}}\right)$ are more than one $\mathrm{eV}$ higher that the ones of Fig. 1, thus making unlikely, at least at high concentration, the formation of the complexes displayed in Fig. 2, as discussed below.

For convenience, in the following discussion, we distinguish, for each type of defect $D$, three different ranges of doping: (1) the highly diluted range $(x \ll 1)$, in which the average distance between different complexes is so high that the complexes can be considered as non-interacting; (2) the interaction range, in which the average distances between randomly distributed complexes allow a non-negligible overlap of the wave-function of different complexes, producing a impurity band (IB) in the gap; and (3) the fully metallic range for concentration greater than the concentration $x_{M}(D)$ at which the IB merges into the

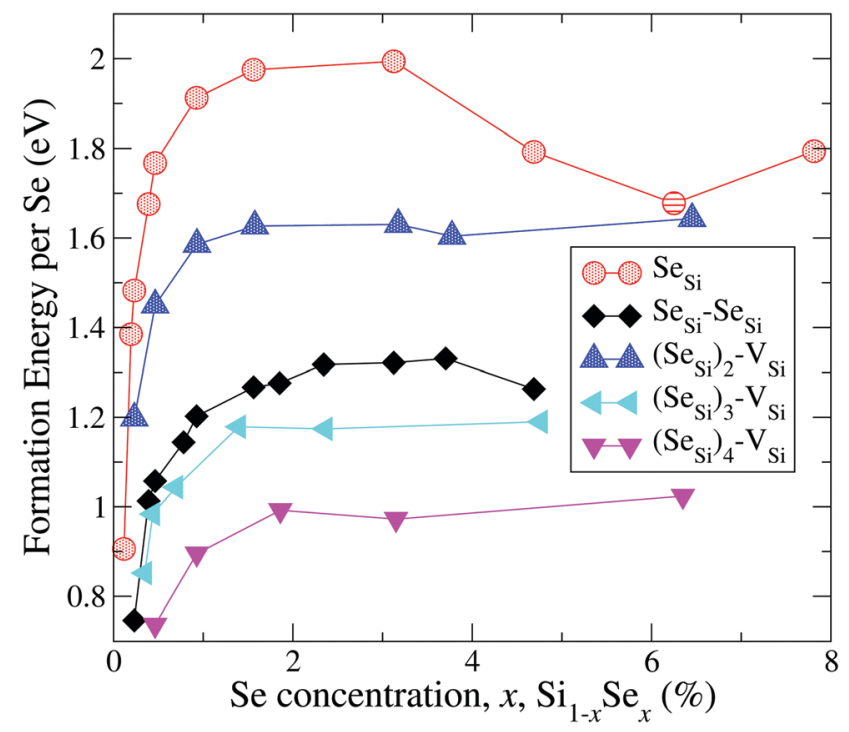

Fig. 1 Formation energy (per atom) of Se substitutional impurities and their complexes with a silicon vacancy. Solid lines are a guide for the eye. 


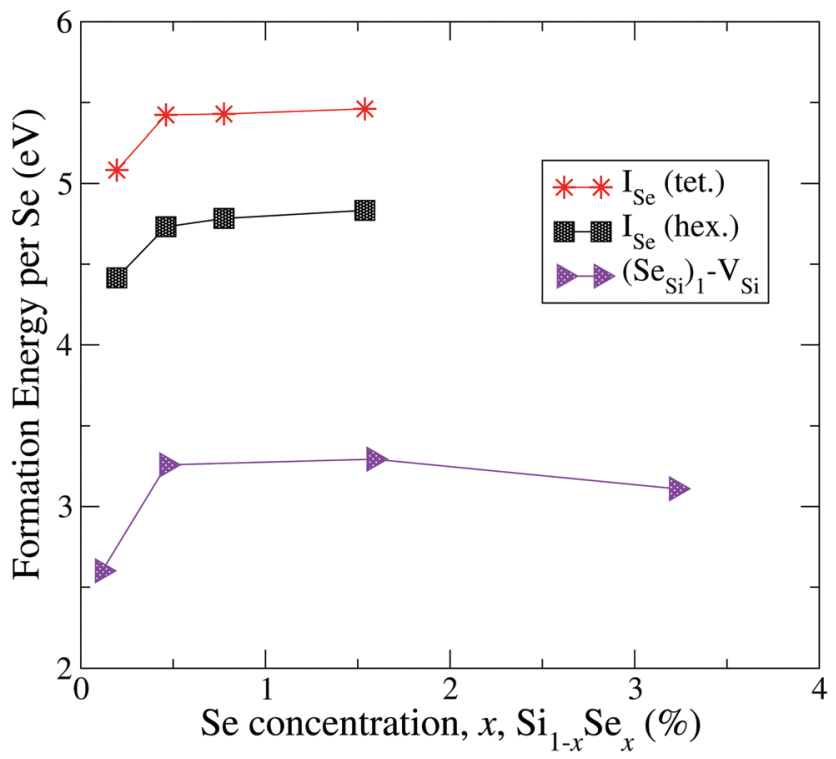

Fig. 2 Formation energy (per atom) of Se interstitials and the complex formed by Se substitutional with a silicon vacancy.

conduction band; in this range the electrons originating from the IB are de-localized in the conduction bands thus contributing to the metallic behaviors.

The $a b$ initio simulation of a highly diluted range, requiring enormous size super-cells, is beyond the scope of the present work, which is focused on the interaction and the metallic ranges corresponding to hyper-doping. Donor complexes in highly diluted range have localized wave-functions, producing energy levels in the bandgap having sharp (zero or negligible) linewidth due to negligible overlap between the wave-functions of different defects. In this range the formation energy is almost equal to the one of the isolated defect, $\Delta E_{D}^{\text {Form }}(0)$.

As the concentration increases (interaction range), the interaction between neighboring complexes produces an IB whose width increases for increasing overlap, and the formation energy steeply increases up to the concentration at which the system presents metallic states delocalized in the whole crystal.

In the fully metallic range, for $x>x_{M}$, the IB is merged into the conduction band providing conduction electrons and eventually contributing to a metallic screening of the impurity complex, and $\Delta E_{D}^{\mathrm{Form}}(x)$ is a smooth function of $x$.

As it will be illustrated in the next section, the different types of complexes, can be divided in two sets: complexes that in the interaction range have an insulating $\mathrm{IB}$, denoted as $D_{\text {ins }}$ and complexes that in the interaction range have a metallic IB, denoted as $D_{\text {met }}$. For the latter complexes, we define $x_{\mathrm{s}}\left(D_{\text {met }}\right)$ as the concentration at which $\Delta E_{D}^{\text {Form }}(x)$ becomes smooth; in general, $x_{\mathrm{s}}\left(D_{\text {met }}\right) \lesssim x_{M}\left(D_{\text {met }}\right)$, the non-equality holds probably as a consequence of the screening effects due to the formation of a metallic band.

In general, for all type of complexes investigated, the formation energy increases monotonically with increasing concentration; $\Delta E_{D}^{\text {Form }}(x)$ is steep in the interaction range (up to $x_{\mathrm{s}}$ for $D_{\text {met }}$ ), while it is rather flat in the metallic range. The decrease of
$\Delta E_{\mathrm{SeSi}}^{\mathrm{Form}}(x)$ for $x>3$ at $\%$ is probably due to the mutual interaction of different $\mathrm{Se}_{\mathrm{S}}$, that above the $x_{M}\left(\mathrm{Se}_{\mathrm{Si}}\right)$ produces a lowering of the formation energy approaching the one of dimers as the concentration increases.

These differences in $\Delta E_{D}^{\text {Form }}(x)$ explain the prevalence of complexes involving substitutional Se with respect to Se interstitials in the metallic range, detected in experimental studies, since according to our simulations the former complexes have lower formation energy in the region where $\Delta E_{D}^{\mathrm{Form}}(x)$ is flat. Although similar differences in $\Delta E_{D}^{\text {Form }}(x)$ can be also noticed in the lower values of $x$ range considered, a local fluctuation of the concentration $\Delta x$, provided by the random distribution of dopants, can cause a significant variation in the formation energy of the complex if $x$ is within the interaction range in which $\Delta E_{D}^{\mathrm{Form}}(x)$ is steep, thus preventing the complexes having the lowest $\Delta E_{D}^{\text {Form }}(x)$ to become the dominant ones.

To better illustrate this concept, we consider two complexes $D_{1}$ and $D_{2}$ for which $\Delta E_{D_{1}}^{\text {Form }}(x)<\Delta E_{D_{2}}^{\text {Form }}(x)$; if $x$ is within the steep range of $\Delta E_{D}^{\text {Form }}(x)$ and a local increase (decrease) $\Delta x_{1}\left(-\Delta x_{2}\right)$ occurs in a region of the crystal where the complexes $D_{1}\left(D_{2}\right)$ are present, this fluctuation can produce $\Delta E_{D_{1}}^{\text {Form }}\left(x+\Delta x_{1}\right)>\Delta E_{D_{2}}^{\text {Form }}\left(x-\Delta x_{2}\right)$.

At variance, if $x$ is within the metallic range, where $\Delta E_{D}^{\text {Form }}(x)$ is smooth, the formation energy is only slightly affected by a local fluctuation of $x$ (in fact, in the smooth range a local fluctuation does not change the inequality $\Delta E_{D_{1}}^{\mathrm{Form}}\left(x+\Delta x_{1}\right)<$ $\left.\Delta E_{D_{2}}^{\text {Form }}\left(x-\Delta x_{2}\right)\right)$, and thus the complexes having lowest $\Delta E_{D}^{\text {Form }}(x)$ are the predominant ones that we expect to detect in an experiment.

The complexes formed by one substitutional Se nearestneighbour (NN) to a Si vacancy, $\mathrm{Se}_{\mathrm{Si}}-\mathrm{V}_{\mathrm{Si}}$, and the Se interstitial, $\mathrm{I}_{\mathrm{Se}}$, in the hexagonal or in the tetrahedral position, have significantly higher formation energy than the other complexes investigated; for $x \gtrsim 0.45$ at $\%$ the formation energy of these complexes is smooth, and consequently, the formation of these complexes is unlikely, explaining the dramatic reduction of interstitial $\mathrm{Se}$ at these concentrations as experimentally detected in Se hyperdoped $\mathrm{Si}^{32,33}$ A similar mechanism has been also found in Te hyperdoped $\mathrm{Si}^{6}$ (by comparing $\Delta E_{D}^{\mathrm{Form}}$ of $\left.\mathrm{I}_{\mathrm{Te}}, \mathrm{Te}_{\mathrm{Si}}, \mathrm{Te}_{\mathrm{Si}}-\mathrm{Te}_{\mathrm{Si}}\right)$.

\section{B Electronic bandstructure}

To analyze the electronic properties of different defects, in Fig. 3 and 4 we display the Density of States (DOS) of the different types of complexes studied for $x \in[0.44-0.46]$ at\%. With this choice of $x$, all types of defects considered have equal or very similar Se concentration thus allowing the comparison between the IB of different complexes.

At this concentration, the IB of $\mathrm{I}_{\mathrm{Se}}$ in the hexagonal position and the IB of $\left(\mathrm{Se}_{\mathrm{Si}}\right)_{3}-\mathrm{V}_{\mathrm{Si}}$ have already been merged with the conduction band, while the IBs formed by the other complexes are within the bandgap originated from the Si gap. $\left(\mathrm{Se}_{\mathrm{Si}}\right)_{4}-\mathrm{V}_{\mathrm{Si}}$ has a shallow metallic IB. At variance, $\mathrm{I}_{\mathrm{Se}}$ in the tetrahedral position and $\left(\mathrm{Se}_{\mathrm{Si}}\right)_{1}-\mathrm{V}_{\mathrm{Si}}$ have metallic IB deep in the bandgap, and both complexes are double acceptors, thus acting as deactivation centers for electronic carriers. According to Fig. 2 

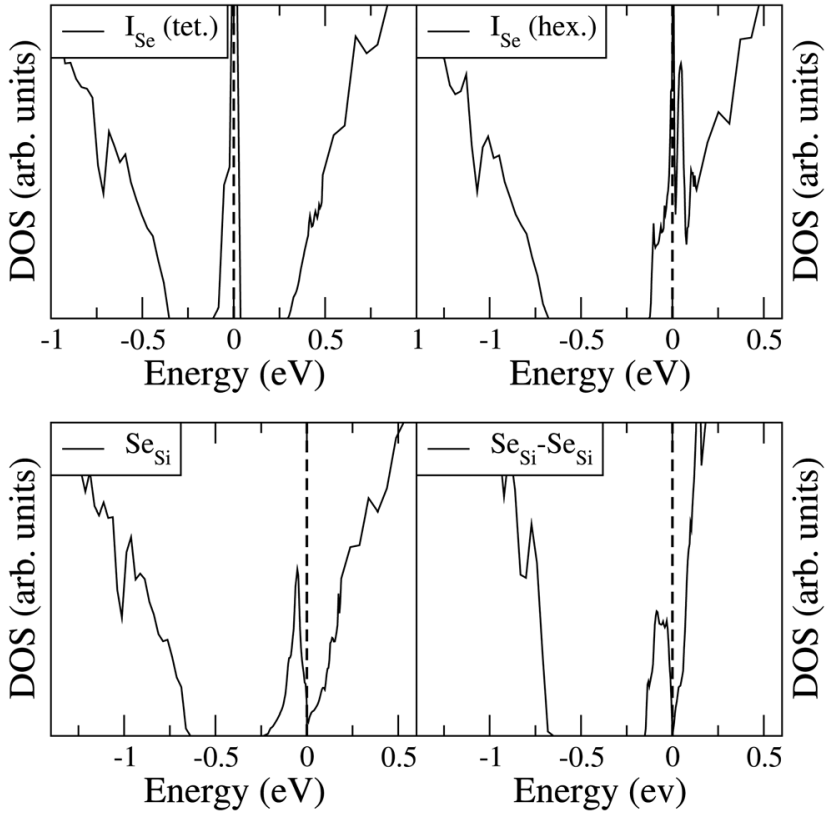

Fig. 3 Electronic DOS of $\mathrm{Si}_{1-x} \mathrm{Se}_{x}$ for Se interstitial in the tetrahedral and hexagonal positions at $x=0.461$ at\%, and for substitutional single $\mathrm{Se}_{\mathrm{Si}}$ and $\mathrm{Se}_{\mathrm{Si}}-\mathrm{Se}_{\mathrm{Si}}$ dimers at $x=0.463$ at\%.
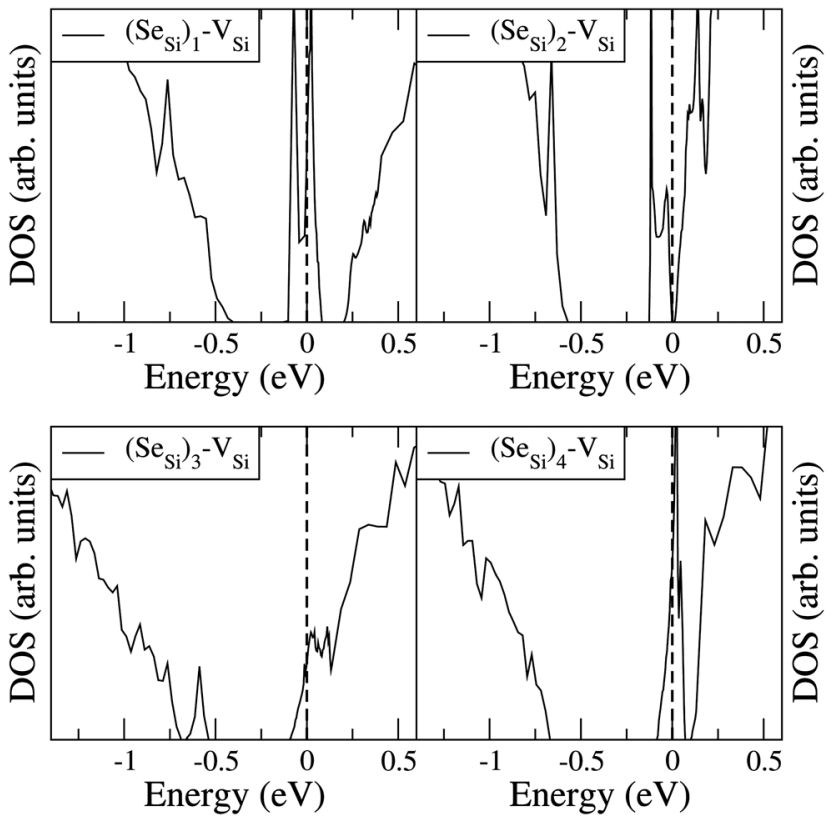

Fig. 4 Electronic DOS of $\mathrm{Si}_{1-x} \mathrm{Se}_{x}$ for $\left(\mathrm{Se}_{\mathrm{si}}\right)_{m}-\mathrm{V}_{\mathrm{Si}}$, with $m=1,2,3$, and 4 at $x \simeq 0.465 ; 0.464 ; 0.438$; and 0.463 at\%, respectively

the formation energy of the latter two complexes for $x \gtrsim 0.45$ at $\%$ is flat, in agreement with the fact that the complexes present metallic behaviors, and thus produces a drastic reduction of undesired deactivation centers for $x>0.45$ at $\%$.

Fig. 3 and 4 show that $\mathrm{Se}_{\mathrm{Si}}, \mathrm{Se}_{\mathrm{Si}_{\mathrm{i}}}-\mathrm{Se}_{\mathrm{Si}}$, and $\left(\mathrm{Se}_{\mathrm{Si}}\right)_{2}-\mathrm{V}_{\mathrm{Si}}$ form insulating IBs, occupied with two electrons per complex.
While the IB filling with two electrons per $\mathrm{Se}_{\mathrm{Si}}$ is simply understood since Se has two more valence electrons than Si thus acting as double donor, the IB filling with two electrons per $\mathrm{Se}_{\mathrm{Si}_{\mathrm{i}}}-\mathrm{Se}_{\mathrm{Si}}$, can be explained as follows: three electrons from each of the two $\mathrm{NN} \mathrm{Se}_{\mathrm{Si}}$ are involved in the bond with the three $\mathrm{NN}$ of the host $\mathrm{Si}$, while two electrons of each $\mathrm{Se}_{\mathrm{Si}}$ are involved in the Se-Se double bond in a similar way to that in $\mathrm{Se}_{2}$ molecule $^{34}$. In fact, according to our simulations the value of the Se-Se bond of $\mathrm{Se}_{2}$ molecule is $2.18 \AA$, quite close ( $\sim 10 \%$ smaller) to the value of the Si-Si bond in bulk $\mathrm{Si}$, which is $2.37 \AA$. This bond filling leaves one unpaired electron for each $\mathrm{Se}_{\mathrm{Si}}$, thus accounting for the fact that $\mathrm{Se}_{\mathrm{Si}}-\mathrm{Se}_{\mathrm{Si}}$ is a double donor in Si i.e. each Se provide one electron to the conduction band). Probably, a similar mechanism can also be invoked for $\left(\mathrm{Se}_{\mathrm{Si}}\right)_{2}-\mathrm{V}_{\mathrm{Si}}$. Consequently, the latter two complexes, each involving two substitutional Se, donate one electron per - Se atom to the conduction band.

The Fermi energy intersects the IB of $\mathrm{I}_{\mathrm{Se}}$ in tetrahedral position and the IB of $\left(\mathrm{Se}_{\mathrm{Si}}\right)_{1}-\mathrm{V}_{\mathrm{Si}}$; each IB is deep in the bandgap and both complexes are double acceptors, thus acting as deactivation centers for electronic carriers. Notice that for $\left(\mathrm{Se}_{\mathrm{Si}}\right)_{1}-\mathrm{V}_{\mathrm{Si}}$ the Fermi energy separates two well resolved peaks in the DOS at the IB, a reminiscence of the separated filled and empty electronic levels of isolated complex, now merged in a metallic IB. As shown in Fig. 2, for these complexes, the concentration $x \simeq 0.45$ at $\%$ corresponds to the flat range of the formation energy, in agreement with the fact that the complexes present metallic behaviors, as discussed above.

Similar results can be deduced from the analysis of the electronic bandstructure. The interested reader can find a detailed discussion of the electronic bands in relation to the electrical properties of the different types of defects in Appendix C.

\section{Geometry of Complexes involving substitutional Se}

In Tables 1 and 2 , for $x \simeq 0.45$ at\%, we display the geometry of fully relaxed complexes involving substitutional Se, reporting the distances $d$ between all the atoms of the complex. In the first columns of the tables, we report the distances $d$ (p.d. $-X$ ) between the point defect (p.d. $=\mathrm{V}_{\mathrm{Si}}, \mathrm{Se}_{\mathrm{Si}}$ ) and the $\mathrm{NN}$ atoms forming the complex $\left(\mathrm{X}=\mathrm{Se}_{\mathrm{Si}}, \mathrm{Si}\right.$, assuming the vacant atom lying at the ideal position in the pristine lattice). In the last columns we report the distances $d(\mathrm{X}-\mathrm{X})$ between the four atoms (6 distances) neighboring $\mathrm{V}_{\mathrm{Si}}\left(\mathrm{Se}_{\mathrm{Si}}\right)$ or between the six $\mathrm{Si}$ (15 distances) $\mathrm{NN}$ to $\mathrm{Se}$ in $\mathrm{Se}_{\mathrm{Si}}-\mathrm{Se}_{\mathrm{Si}}$. Note that while the distances correspond to relaxed positions, the symbol $\mathrm{NN}^{n}$

Table 1 Distances between atoms within the tetrahedron containing the complex defect. All the values are given in angströms, in parenthesis the number of equal bonds (NN) or equal distances $\left(\mathrm{NN}^{2}\right)$

\begin{tabular}{lllllll}
\hline Complex & Conc. (\%) & $d\left(\mathrm{~V}_{\mathrm{Si}^{-}}-\mathrm{Se}\right)$ & $d\left(\mathrm{~V}_{\mathrm{Si}}-\mathrm{Si}\right)$ & $d(\mathrm{Se}-\mathrm{Se})$ & $d(\mathrm{Se}-\mathrm{Si})$ & $d(\mathrm{Si}-\mathrm{Si})$ \\
\hline$\left(\mathrm{Se}_{\mathrm{Si}}\right)_{1}-\mathrm{V}_{\mathrm{Si}}$ & 0.465 & $2.30(1)$ & $2.09(3)$ & - & $3.63(3)$ & $3.37(3)$ \\
$\left(\mathrm{Se}_{\mathrm{Si}}\right)_{2}-\mathrm{V}_{\mathrm{Si}}$ & 0.464 & $2.25(2)$ & $2.06(2)$ & $3.69(1)$ & $3.52(4)$ & $3.35(1)$ \\
$\left(\mathrm{Se}_{\mathrm{Si}}\right)_{3}-\mathrm{V}_{\mathrm{Si}}$ & 0.438 & $2.29(3)$ & $2.12(1)$ & $3.74(3)$ & $3.61(3)$ & - \\
$\left.(\mathrm{Se})_{\mathrm{Si}}\right)_{4}-\mathrm{V}_{\mathrm{Si}}$ & 0.463 & $2.33(4)$ & - & $3.80(6)$ & - & - \\
Near. neigh. shell & \multicolumn{2}{c}{$\mathrm{NN}$} & & $\mathrm{NN}^{2}$ &
\end{tabular}


Table 2 Distances between atoms within the tetrahedron (two tetrahedra) containing the $\mathrm{Se}_{\mathrm{Si}}\left(\mathrm{Se}_{\mathrm{Si}}-\mathrm{Se}_{\mathrm{Si}}\right)$ defect (complex). All the values are given in angströms, in parenthesis the number of equal bonds (NN) or equal distances $\left(\mathrm{NN}^{n}, n \geq 2\right)$

\begin{tabular}{|c|c|c|c|c|c|c|c|}
\hline Complex & $\begin{array}{l}\text { Conc. } \\
(\%)\end{array}$ & $d(\mathrm{Se}-\mathrm{Se})$ & $d(\mathrm{Se}-\mathrm{Si})$ & & $d(\mathrm{Si}-\mathrm{Si})$ & & \\
\hline $\mathrm{Se}_{\mathrm{Si}}$ & 0.463 & - & $2.57(4)$ & 4.19 (12) & - & - & - \\
\hline $\mathrm{Se}_{\mathrm{Si}}-\mathrm{Se}_{\mathrm{Si}}$ & 0.463 & 3.15 (1) & $2.44(3)$ & $4.37(3)$ & $4.12(6)$ & $4.82(6)$ & $6.34(3)$ \\
\hline Near. neig & h. shell & $\mathrm{NI}$ & & NI & $\mathrm{N}^{2}$ & $\mathrm{NN}^{3}$ & $\mathrm{NN}^{4}$ \\
\hline
\end{tabular}

labels the atoms according to the ideal positions in the pristine lattice in the $n$-th $\mathrm{NN}$ shell from the p.d. The $\left(\mathrm{Se}_{\mathrm{Si}}\right)_{m}-\mathrm{V}_{\mathrm{Si}}$ complexes show an inward relaxation of Se toward the vacancy. At variance, for $\mathrm{Se}_{\mathrm{Si}}$ the relaxed $d(\mathrm{Se}-\mathrm{Si})$ is $8 \%$ isotropically larger than the simulated ideal Si-Si bond ( $2.37 \AA$ ̊).

Since they can be measured by Rutherford back-scattering spectrometry in channeling geometry (RBS-C), we report, for $x \simeq 0.45 \mathrm{at} \%$, the atomic displacements of Se from the ideal position in the pristine lattice. While for $\left(\mathrm{Se}_{\mathrm{Si}}\right)_{m}-\mathrm{V}_{\mathrm{Si}}, m=1,3$, or 4 , the atomic displacement is very small (about $0.06,0.08$, and $0.04 \AA$ for $m=1,3$, and 4, respectively) for $\mathrm{Se}_{\mathrm{Si}^{-}}-\mathrm{Se}_{\mathrm{Si}}$ and $\left(\mathrm{Se}_{\mathrm{Si}}\right)_{2}-$ $\mathrm{V}_{\mathrm{Si}}$ the Se displacement is $0.39 \AA$, and $0.12 \AA$, respectively, representing a fingerprint of the peculiar bond formed by couples of chalcogen donors in Si. Similar values of Se displacements are obtained for other concentrations.

\section{Discussion}

We briefly discuss our finding in view of possible applications of chalcogen hyperdoped $\mathrm{Si}$ as the building block in broad band infra-red detectors or intermediate band photo-voltaics. Both applications are based on the formation of a relatively shallow IB.

At room temperature - the usual device working temperature all IB electrons are thermally excited in the conduction band, while the absorption of infra-red radiation (or of sunlight to photo-generate electric current for photo-voltaics) is produced by the excitation of valence-band electrons into the IB.

Our goal is to determine the best doping range for these applications.

Assuming a uniform random distribution of substitutional Se in the lattice sites, the complexes involving three or more donors are extremely unlikely (for a quantitative analysis see Appendix B). The complexes of Fig. 1 involving up to two Se have insulating IB, for these $D_{\text {ins }}$ defects we found

$$
\begin{aligned}
0.46 \mathrm{at} \% \cong x_{M}\left(\mathrm{Se}_{\mathrm{Si}}\right) & <x_{M}\left(\left(\mathrm{Se}_{\mathrm{Si}}\right)_{2}-\mathrm{V}_{\mathrm{Si}}\right)<x_{M}\left(\mathrm{Se}_{\mathrm{Si}}-\mathrm{Se}_{\mathrm{Si}}\right) \\
& \cong 0.93 \mathrm{at} \%
\end{aligned}
$$

A first technological requirement is to maximize the electrons in conduction band by drastically reducing the electrical deactivation complexes.

According to the above analysis for $x \gtrsim 0.46$ at $\%$ the system presents a dramatic reduction of the deactivation complexes $\mathrm{I}_{\mathrm{Se}}$ and $\left(\mathrm{Se}_{\mathrm{Si}}\right)_{1}-\mathrm{V}_{\mathrm{Si}}$.
Notice that, for $x \gtrsim 0.46$ at $\%$, the other types of complexes having a metallic IB don't act as acceptors, because they have (1) a shallow IB, which is fully ionized at room temperature (as $\left(\mathrm{Se}_{\mathrm{Si}}\right)_{4}-\mathrm{V}_{\mathrm{Si}}$ at $x=0.46$ at\%); or they have (2) the IB merged into the conduction band $\left(x_{M}\left(\left(\mathrm{Se}_{\mathrm{Si}}\right)_{3}-\mathrm{V}_{\mathrm{Si}}\right)<0.43\right.$ at\%).

This condition determines the lower limit of the best doping range, $x_{\mathrm{inf}}=x_{M}\left(\mathrm{Si}_{\mathrm{Se}}\right)$.

A second technological requirement is the presence of a broad IB in the gap. As shown in Fig. 3 and 4 different types of complexes have different IB-widths and different IB-centers, their DOS overlap only partially in the energy interval corresponding to the IBs; these overlap of the DOS contributes to further increase the total width of the resulting IB (which is the sum of the IB of all types of defects present in the sample), at least up to the concentration $x_{M}\left(\mathrm{Se}_{\mathrm{Si}}-\mathrm{Se}_{\mathrm{Si}}\right)$ that corresponds to the greater $x_{M}$ among the donor complexes that have nonnegligible probability to be present in the sample.

The highest $x_{M}$ dictates the upper limit of the best concentration range since it determines the extinction of the IB due to the merging into the conduction band. So, we chose $x_{\text {sup }} \equiv$ $x_{M}\left(\mathrm{Se}_{\mathrm{Si}}-\mathrm{Se}_{\mathrm{Si}}\right) \simeq 0.93 \mathrm{at} \%$, since for $x>x_{\text {sup }}$ we expect that IB of the large majority of Se complexes are merged into the conduction band.

Thus, we suggest that the optimal doping values for intermediate IB application ranges between $x_{\text {inf }}(\simeq 0.46$ at\%), and $x_{\text {sup }}(\simeq 0.93$ at\%); in this range in which a shallow IB is present and is providing the maximum carrier density per donor since the electrical deactivating defects are drastically reduced.

The variation of the dopant concentration within the optimal range provides a tunable mechanism to modify the IB minimum, according to the needs of the electronic industry.

Finally, some considerations about the estimation of $x_{\mathrm{c}}$ within SCM. Neglecting for simplicity $D_{\text {met }}$ complexes, if only one type of defect $D$ is present, the critical temperature corresponds to $x_{M}(D)$. If more than one type of complex is present, what is the critical concentration $x_{\mathrm{c}}$ at which the IMT occurs? By using a simple model in which the complex wavefunction is localized around the impurity and the IB is formed by a tight-binding like form, we propose the formula to evaluate $x_{\mathrm{c}}$ from the values $x_{M}$ obtained by first principles:

$$
\frac{1}{x_{\mathrm{c}}}=\sum_{n} \frac{c_{n}}{x_{M}\left(D_{n}\right)}
$$

where $c_{n}$ is the number of Se forming complexes of type $D_{n}$ divided by the total number of Se. Clearly, $\sum_{n} c_{n}=1$ and $n$ ranges over all different types of complexes.

A simple estimation of most probable defects (assuming a limited mobility of random distributed Se combined with thermal weights, see also Appendix B) gives $x_{\mathrm{c}}=0.50$ at $\%$. The $x_{\mathrm{c}}$ value is mainly determined by $x_{M}$ of the $\mathrm{Se}_{\mathrm{Si}}$ population $\left(x_{M} \simeq 0.46, c_{n} \sim 84 \%\right)$ with respect to the $\mathrm{Se}_{\mathrm{Si}}-\mathrm{Se}_{\mathrm{Si}}$ one $\left(x_{M} \simeq\right.$ $\left.0.93, c_{n} \sim 16 \%\right)$. Our results suggest that the presence of different complexes may increase the IB broadening thus affecting the conductivity of the system, the $x_{\mathrm{c}}$, and the sharpness of the IMT as a function of $x$. 


\section{Conclusions}

In summary, by first principles simulations, we enlighten the microscopic mechanisms responsible for the removal of electrical deactivation defects in Se hyperdoped Si. We studied the formation energy as a function of the dopant concentration and the electronic and structural properties of different Se complexes to identify the optimal doping range, in which the donor density can be tuned to engineering the width and the shallowness of the IB for intermediate IB applications. Our findings can be extended to silicon hyperdoped with other chalcogen impurities, paving the way toward an intermediate IB electronics of nanometer size.

\section{Conflicts of interest}

There are no conflicts to declare.

\section{Appendix}

\section{Appendix A: Computational details}

We performed our first principles calculations of structural and electronic properties of Se hyperdoped silicon within the framework of Density Functional Theory (DFT) by solving the Kohn-Sham equations through the plane-wave pseudopotential approach as implemented in QUANTUM ESPRESSO (QE) opensource package ${ }^{35}$. In our simulations we used ultra-soft pseudopotentials ${ }^{36,37}$ in the separable form introduced by Kleinmann and Bylander ${ }^{38}$, generated using a Perdew-BurkeErnzerhof $(\mathrm{PBE})^{39}$ exchange correlation functional. For metallic systems, i.e. for defects having a metallic IB or for defects having an insulating IB at concentration above the Insulator to Metal Transition (IMT) threshold $\left(x>x_{M}\right)$, we used smearing techniques with a Gaussian broadening of $1 \mathrm{mRy}$. We choose a 40-Ry cutoff radius for the electronic valence wave function and 400-Ry cutoff radius for the charge density. The computed lattice parameter of bulk Si is $a_{\mathrm{L}}=5.475 \AA$ (while $a_{\mathrm{L}}=5.431 \AA$ is the experimental value, from ref. 40).

To simulate the hyperdoped silicon we used the super-cell (SC) method, consisting in the use of large simulation cells containing Se defects. We fully exploit the cubic symmetry of the silicon lattice by building super-cells with different cubic symmetry to increase for each type of defect the set of different doping concentration considered, maintaining the super-cells within a size that can be computationally affordable by our first principles techniques. For this purpose, by using periodic (i.e., Born-von Karman) boundary conditions, we considered simulation cells of different sizes, each containing a different type of Se point defect or Se complex as detailed in the following.

Bulk silicon presents the diamond structure constituted by a face center cubic (fcc) lattice plus a two atom basis. The fcc primitive lattice vectors $\vec{a}_{i}^{\text {fcc }}(I=1,2$, or 3$)$, can be repeated $M$-times, with $M$ a positive integer, to form super-cell of fcc symmetry, denoted fcc- $M M M$, composed of $M^{3}$ unit cells. Explicitly, the super-cell lattice vectors are $\vec{a}_{i}^{\text {sc }} \equiv M \vec{a}_{i}^{\text {fcc }}(i=1$, $2,3)$, where the same integer $M$ is multiplied by each primitive lattice vector to ensure uniform and isotropic dopant distribution, when the super-cell contain one point defect or a isotropic complex as $\left(\mathrm{Se}_{\mathrm{Si}}\right)_{4}-\mathrm{V}_{\mathrm{Si}}$, while it is true with a good approximation if the defect is non-isotropic. Since the unit cell contains 2 atoms, the repetition of the unit cell according a fcc lattice provides super-cells having size $2 M M M$ (i.e. 16, 54, 128, 250, 432, 686,..., for $M=2,3,4,5,6,7, \ldots)$. Since different types of cubic symmetries are possible, a different choice of cubic symmetry provides different super-cell sizes. The conventional unit cell of $\mathrm{Si}$ is a face centered cube with 8 atoms; we considered the conventional cell, as the unit cell of a simple cubic (sc) lattice (whose primitive lattice vectors coincide with the three orthogonal side of the conventional cube), whose 8 atoms unit-cell can be used to build super-cell of type sc- $M M M$, having size $8 M M M$ (i.e., $64,216,512,1000, \ldots$, for $M=2,3,4,5, \ldots$ ), in an analogous way as done for the fcc lattice (by replacing fcc label with sc in the definition of $\left.\vec{a}_{i}^{\mathrm{SC}}\right)$. In a similar way, a sc- $(2 M)(2 M)(2 M)$ super-cell can be seen as body centered cubic (bcc) super-cell containing $\frac{8(2 M)^{3}}{2}=32 \times M^{3}$ atoms (i.e. $32,256,864, \ldots$ for $M=1,2,3, \ldots$ ). The use of All Compatible Symmetries (ACS), in the procedure to build the super-cell (in the present case we use All types of Cubic Symmetries) allows to arrange the doping complexes in a periodic repeated lattice of cubic symmetry (sc, fcc or bcc). This trick significantly increases the number of supercells of different sizes, and is particularly useful, as in our case, in first principle simulations in which one should limit the computational effort to super-cells having less than one thousand atoms, to study complexes composed of different number of dopants, whose electronic properties should be computed at similar dopant concentrations, for comparison.

If only one complex is placed in the super-cell, this technique ensure an uniform distribution of dopants, and by considering the interaction of one complex with its periodic repeated images, each complex can experience a different configuration and different number of nearest neighbour complexes $(6,12$, and 8), for each of the three cubic lattice considered (sc, fcc, bcc, respectively). The regularity of the formation energy as a function of dopant concentration (in Fig. 1 and 2 of the main text, in which the data are obtained by using ACS) suggests that the formation energy is relatively non-sensible on the specific dopant arrangement according to the different cubic system used. This fact, suggests that our results, taken with the ACS technique, are, at least partially, rather unaffected by the disorder effect due to the random distribution of the complexes in a real system. The biggest super-cell considered is the one containing 864 atoms used to simulate the lowest Se concentrations considered (note that $x=0.463$ at $\%$ used to compute the DOS in the bottom-right panel of Fig. 4 is the lowest concentration taken into account to simulate ( $\left(\mathrm{Se}_{\mathrm{Si}}\right)_{4}-\mathrm{V}_{\mathrm{Si}}$, corresponding to 863 atoms).

Thus the ACS distribution of dopants allows us to simulate a great variety of dopant concentrations, as required, due to the different types of complexes considered which can be formed by one up to 4 Se atoms. For each complex, by varying the size of the supercell, we simulate different concentrations of impurities (in our case, the Se concentration ranges from 
approximately 0.12 at $\%$ up to to 7.8 at $\%$ for $\mathrm{Se}_{\mathrm{Si}}$ the defect with the widest concentration range).

In our study, we adopt a single complex model (SCM), in which we assume that only one type of complex is present, and formation energy results from interaction of the complex with the same type of complex in the super-cell if more than one complex are placed in it, and with defect images due to the periodic boundary condition adopted.

This approximation is largely employed in first principles simulation of (hyper-)doped semiconductors (see e.g. ref. 16, 25, 27 and 28, to cite only a few) since combine affordable super-cell size with reliable prediction of formation energy in comparison with experimental data (see also ref. 6 and 29).

So in the hyperdoping regime considered in the present work, the formation energy of a complex in a system where different types of complexes are present, is approximated to the one of a system in which only one type of complex is present. The SCM is expected to provide a qualitative estimation of properties like the formation energy of defect types representing a fraction of the total defect population, while it is expected to provide a reliable quantitative estimation of the complexes representing the large majority of defects present in the sample.

The formation energy of each complex is computed per singe Se atom forming the complex, to allow a direct comparison of the formation energy of complexes composed of a different number of Se atoms.

We mention that, motivated by the decrease of the formation energy of $\mathrm{Se}_{\mathrm{Si}}$ in the range between $x \in[3,6]$ at\% (discussed in the main text), we performed our simulation of this type of defect up to $x=7.8$ at $\%$. The results for this huge range of doping are reported for completeness. However, at this extreme hyperdoping regime we expect that disorder effects and/or phase separation in experimental samples can occur.

The sampling of electronic states over the Brillouin zone was performed by special points techniques by using $2 \times 2 \times 2$ Monkhorst-Pack grid ${ }^{41}$ for a super-cell having cubic symmetry with 216 atoms. For super-cell of different size the MonkhorstPack grid was modified accordingly to ensure an uniform sampling grid in the Brillouin zone.

In the silicon lattice site a single vacancy $\mathrm{V}_{\mathrm{Si}}$ was created and decorated with $\left(\mathrm{Se}_{\mathrm{Si}}\right)_{m}$, with $m=1-4$ atoms in substitutional nearest neighbors positions to create the complex $\left(\mathrm{Se}_{\mathrm{Si}}\right)_{\mathrm{m}}-\mathrm{V}_{\mathrm{Si}}$. The substitutional (interstitial) Se are placed in the corresponding lattice site (position) of pristine Si.

After structural and atomic relaxation we performed the calculation of formation energy and other electronic properties (electronic bandstructure and/or the density of states (DOS)). Electronic occupation of the IB is obtained by the integration of the DOS. The Se concentration for all complexes refers to the percentage concentration of Se atoms with respect to the total amount of $\mathrm{Si}$ plus Se atoms in the super-cell. At a given concentration, to simulate the different types of complexes the size of the super-cell (i.e. the total number of atomic sites) and/or the number of Se atoms in the supercell are arranged accordingly, to obtain the stated Se concentration.
The formation energy is computed according to eqn (1) of the main text; the more natural choice for $\mu_{\mathrm{Si}}$ corresponds to the chemical potential of bulk silicon (usually referred as the Si-rich chemical potential), evaluated according to the standard procedure (see, e.g., ref. 40 and 42 ) by taking the total energy of the unit cell computed by DFT divided by the number of atoms contained in the unit cell. Our choice for $\mu_{\mathrm{Se}}$ corresponds to the Se chemical potential of bulk $\mathrm{SiSe}_{2}$ in equilibrium with bulk silicon. So, $\mu_{\mathrm{Si}}$ is evaluated by subtracting the chemical potential of bulk Si from the total energy of the $\mathrm{SiSe}_{2}$ unit cell computed by DFT and dividing the result by 2 . Note that, for the present case, the choice of the chemical potential of Se simply shifts the zero of the energy, i.e., the vertical axis in Fig. 1 and 2 in the main text.

We focused our attention on complexes involving substitutional Se, motivated by the experimental evidence that at high chalcogen concentration in Si i.e. concentration comparable or higher than $x_{\mathrm{c}}$, the critical concentration at which the IMT occurs) the substitutional impurities are the predominant type of defect (at least for $\mathrm{Se}^{32,33}$ and $\mathrm{Te}^{6}$ ), a fact that can be explained on the basis of first principles calculations by the significantly higher formation energy of chalcogen in interstitial position than the substitutional ones, as shown in ref. 6 for Te interstitials compared to the substitutional single Te and Te dimer and in the present study for Se interstitial compared to all types of Se substitutional complexes investigated.

\section{Appendix B: What are the most probable defects?}

The relative concentrations of the defects $I_{\mathrm{Se}}$, and of the complex $\left(\mathrm{Se}_{\mathrm{Si}}\right)_{1}-\mathrm{V}_{\mathrm{Si}}$ (i.e. the ones displayed Fig. 2 of the main text) can be safely neglected, at least in the metallic range, due to the unfavorable formation energy, which is more than $1.5 \mathrm{eV}$ higher than the ones of the $\mathrm{Se}_{\mathrm{Si}}$ (and to the other types of complexes displayed of Fig. 1 of the main text), thus favoring the collocation of Se in the substitutional position and penalizing the creation of $\mathrm{V}_{\mathrm{Si}}$ in the NN sites to a single Se.

In contrast, the relative concentration of complexes of the type displayed Fig. 1 is a more delicate balance between the thermal processes related to temperatures involving the formation energy of different complexes, and the kinematic processes related to the limited Se mobility to account a formation of a compound whose se concentration is beyond the solid solubility limit.

For the calculation of the relative concentration of different types of complexes present in Se hyperdoped $\mathrm{Si}$, we propose the following model according to the practical recipe described below.

We assume a random distribution of dopants whose probability can be evaluated by statistical methods (see e.g., ref. 25, a justification of this assumption is also provided in Appendix D), and we consider a region of limited size (typically including few tens lattice sites). To compute the relative concentration of different types of complex, we assign to each complex a thermal Boltzmann weight according to the formation energies computed for the given complex if the number of dopants present in this region is compatible with the number of dopants forming the complex. 
We illustrate our procedure by applying our model to the results obtained in the main text. We assume that the dopants are randomly distributed at the silicon lattice sites with probability $x=\frac{N_{\mathrm{Se}}}{N_{\mathrm{Si}}}$, where $N_{\mathrm{Se}}$ and $N_{\mathrm{Si}}$ are the total number of Se and Si atoms present in the system, respectively (or in the supercell, if, as in our case, one applies periodic boundary conditions). In our model, we consider a lattice site occupied by a dopant (Se) and the lattice sites of the neighbor shells surrounding the dopant, up to the $n_{\max }$-th shells having the dopant (Se) atom at the center. We call this region the $n_{\text {max }}$-sphere.

If Se are randomly distributed at the Si lattice sites, $x$ is the probability that the lattice site is occupied by a Se, while $\chi \equiv 1-x$ is the probability that the lattice site is occupied by a Si. Let $z_{k}$ be the number of lattice sites in the $k$-th shell, we define:

$$
S_{n_{\max }} \equiv \sum_{k=1}^{n_{\max }} z_{k}
$$

The probability that the Se at the center of the shells is surrounded by $m$-Se placed randomly in the lattice sites up to the $n_{\text {max }}$-shell is

$$
p_{m+1}^{(0)}=\chi^{S_{n_{\max }}}\left(\frac{1}{\chi}-1\right)^{m} \frac{S_{n_{\max }} !}{m !\left(S_{n_{\max }}-m\right) !}
$$

So $p_{m^{\prime}}^{(0)}$ is the probability of finding $m^{\prime}$-Se (including the Se at the center) randomly placed in the $n_{\max }$-sphere.

With eqn (5) we can easily evaluate the probability, $p_{m>2}^{(0)}$ that within the first three neighboring shells (i.e., in the $n_{\max }{ }^{-}$ sphere, with $n_{\max }=3$ ) there are more than two Se (including the Se at the center). At $x=0.45$ at $\%$ the probability is $p_{m>2}^{(0)}=0.8668 \times$ $10^{-2}$ (hereafter $p_{m}^{(0)}$ will be normalized to unity, if not explicitly stated otherwise), while at $x=0.95$ at $\%$ the probability is $p_{m>2}^{(0)}=$ $0.2897 \times 10^{-1}$. So, according to our model, within the concentration range of interest, the probability of forming $\left(\mathrm{Se}_{\mathrm{Si}}\right)_{3}-\mathrm{V}_{\mathrm{Si}}$ and $\left(\mathrm{Se}_{\mathrm{Si}}\right)_{4}-\mathrm{V}_{\mathrm{Si}}$ is less than $3 \%$. So, we neglect the possibility of forming the complexes which involve three or four Se, and we focus on our estimation of the complexes involving one or two dopants.

In our model, the formation of a complex $D$ having a formation energy $\Delta E_{D}^{\text {Form }}$ can occur at temperature $T$ according to a probability proportional to the Boltzmann distribution, if and only if the number of dopants which are randomly placed in the $n_{\max }$-sphere is equal to or greater than the number of dopants forming the complex. We re-call that $p_{m}^{(0)}$ is the probability of finding $m$-Se randomly placed in the $n_{\max }$-sphere, and, according to the above consideration, we chose for simplicity $m \leq 2$. The probability of finding a defect $D_{n}$ constituted by $n$-dopants in the $n_{\max }$-sphere containing $m$-Se is

$$
p_{m}\left(D_{n}\right)= \begin{cases}p_{m}^{(0)} \cdot \frac{1}{Z_{m}} \mathrm{e}^{-\Delta E_{D_{n}}^{\mathrm{Form}} / k_{\mathrm{B}} T} & n \leq m \\ 0 & n>m\end{cases}
$$

where $k_{\mathrm{B}}$ is the Boltzmann constant, and $Z_{m} \equiv \sum_{D_{n}}^{n \leq m} \mathrm{e}^{-\Delta E_{D_{n}}^{\text {Form }} / k_{\mathrm{B}} T}$ is the partition function with $n$ that runs over all the distinct types of defects $D_{n}$ with $n \leq m$. The relative concentration of defect $D_{n}$ is given by $p\left(D_{n}\right)=\sum_{m} p_{m}\left(D_{n}\right)$.

We now apply our model to evaluate the probability of different complexes within the optimal doping range for intermediate band applications, as reported in the main text.

In our estimation we choose $x=0.75$ at $\%, n_{\max }=3$, and $m \leq 2$. For this choice of parameters, the probability to find only one Se within the $n_{\max }$-sphere is $p_{1}{ }^{(0)}=0.8214$, while the probability of finding two Se within the $n_{\max }$-sphere is $p_{2}{ }^{(0)}=0.1621$. The probability of finding one $\mathrm{Se}_{\mathrm{Se}}$ in a $n_{\max }$-sphere containing only one random Se is obviously $p_{1}\left(\mathrm{Se}_{\mathrm{Si}}\right)=p_{1}{ }^{(0)}$, according to eqn (6). Less trivial is the case of two random se present in the $n_{\max }$-sphere. Within the $n_{\text {max }}$-sphere the two Se can arrange according to the Boltzmann distribution to the three allowed configurations: two single $\mathrm{Se}_{\mathrm{Si}}$, or one $\mathrm{Se}_{\mathrm{Si}}-\mathrm{Se}_{\mathrm{Si}}$ dimer, or one $\left(\mathrm{Se}_{\mathrm{Si}}\right)_{2}-\mathrm{V}_{\mathrm{Si}}$ complex. According to eqn (6) where $Z_{2}=$ $\sum_{D} \mathrm{e}^{-\Delta E_{D}^{\text {Form }} / k_{\mathrm{B}} T}$ is the partition function and $D \in\left[\mathrm{Se}_{\mathrm{Si}} ; \mathrm{Se}_{\mathrm{Si}}-\mathrm{Se}_{\mathrm{Si}}\right.$; $\left.\left(\mathrm{Se}_{\mathrm{Si}}\right)_{2}-\mathrm{V}_{\mathrm{Si}}\right]$. With the $p^{(0)}$ parameters reported above, and the formation energy reported in Table 3 we evaluate the relative concentration of the complexes: $\mathrm{Se}_{\mathrm{Si}}, \mathrm{Se}_{\mathrm{Si}}-\mathrm{Se} \mathrm{Si}_{\mathrm{Si}},\left(\mathrm{Se}_{\mathrm{Si}}\right)_{2}-\mathrm{V}_{\mathrm{Si}}$, the results for the relative concentration $p(D)$ are displayed in the same table. As reported in the table, according to our model the large majority of the complexes is constituted by $\mathrm{Se}_{\mathrm{Si}}$, and $\mathrm{Se}_{\mathrm{Si}}-\mathrm{Se}_{\mathrm{Si}}$. All other types of complexes contribute less than $3 \%$ to the relative concentration for all types of complexes.

The choice of the temperature in eqn (6) is rather arbitrary. To evaluate the relative concentrations displayed in Table 3 we take $T=1687 \mathrm{~K}$, i.e. equal to Si melting point, which corresponds to the maximum value that the sum of the concentration of all other complexes different from $\mathrm{Se}_{\mathrm{Si}}$, and $\mathrm{Se}_{\mathrm{Si}}-\mathrm{Se}_{\mathrm{Si}}$ can assume (within our model and the choice of the parameters different than $T$ ). For lower temperatures, $T<1687 \mathrm{~K}$, the sum of the relative concentrations of all other complexes should be lower.

Therefore, to evaluate the critical concentration, $x_{\mathrm{c}}$, at which the IMT occurs, we limited ourselves to consider the $\mathrm{Se}_{\mathrm{Si}}$, and $\mathrm{Se}_{\mathrm{Si}}-\mathrm{Se}_{\mathrm{Si}}$ populations (re-normalizing the relative concentration considering only these two types of complexes). We obtained $x_{\mathrm{c}}$ $=0.50$ at $\%$. The $x_{\mathrm{c}}$ value is mainly determined by $x_{M}$ of $\mathrm{Se}_{\mathrm{Si}}$ population $\left(x_{M} \simeq 0.46, c_{n} \sim 84 \%\right)$ with respect to $\mathrm{Se}_{\mathrm{Si}}-\mathrm{Se}_{\mathrm{Si}}$ one $\left(x_{M} \simeq 0.93, c_{n} \sim 16 \%\right)$. This result, reported in the main text, is quite intuitive: complexes involving three or more se are penalized by the low probability to find three or more se close enough to form a complex, while the formation of the complex

Table 3 Relative concentration of $\mathrm{Se}$ complexes in $\mathrm{Si}_{1-x} \mathrm{Se}_{x}$ at $x=$ 0.75 at\%. The values of the formation energy used in the evaluation of the complex concentration are reported in the first column

\begin{tabular}{llc}
\hline Complex & $\Delta E_{D}^{\text {Form }}(\mathrm{eV})$ & Concentration (\%) \\
\hline $\mathrm{Se}_{\mathrm{Si}}$ & 1.90 & 82.21 \\
$\left(\mathrm{Se}_{\mathrm{Si}}\right)_{2}-\mathrm{V}_{\mathrm{Si}}$ & 1.59 & 0.56 \\
$\mathrm{Se}_{\mathrm{Si}}-\mathrm{Se}$ & 1.11 & 15.58 \\
Other complexes & - & 1.65
\end{tabular}


$\left(\mathrm{Se}_{\mathrm{Si}}\right)_{2}-\mathrm{V}_{\mathrm{Si}}$ is energetically unfavored with respect to $\mathrm{Se}_{\mathrm{Si}}-\mathrm{Se}_{\mathrm{Si}}$ by the higher formation energy.

To provide an intuitive, albeit approximate, picture of the mechanism involved in our model we assume that, in the silicon crystal which constitutes the experimental sample, the Se atoms have a very limited mobility, remaining close (says within a NN-distance) to the lattice sites where the Se atoms were originally located by the random distribution (obviously, the model can be generalized to include the moving to $N N^{n}$ lattice sites). At variance, we assume that the Si atoms have very high mobility, since the formation of Si vacancies is assumed to occur according to a Boltzmann factor. This assumption can be justified, at least in part, by the fact that a Si atom has a smaller covalent radius than a Se atom.

\section{Appendix C: Electronic bandstructure}

To prove our estimation of $x_{M}$ for $\mathrm{Se}_{\mathrm{Si}}$ and $\mathrm{Se}_{\mathrm{Si}}-\mathrm{Se}_{\mathrm{Si}}$, in Fig. 5 we display the bandstructure of single $\mathrm{Se}_{\mathrm{Si}}$ at $x=0.46$ at\%, while in

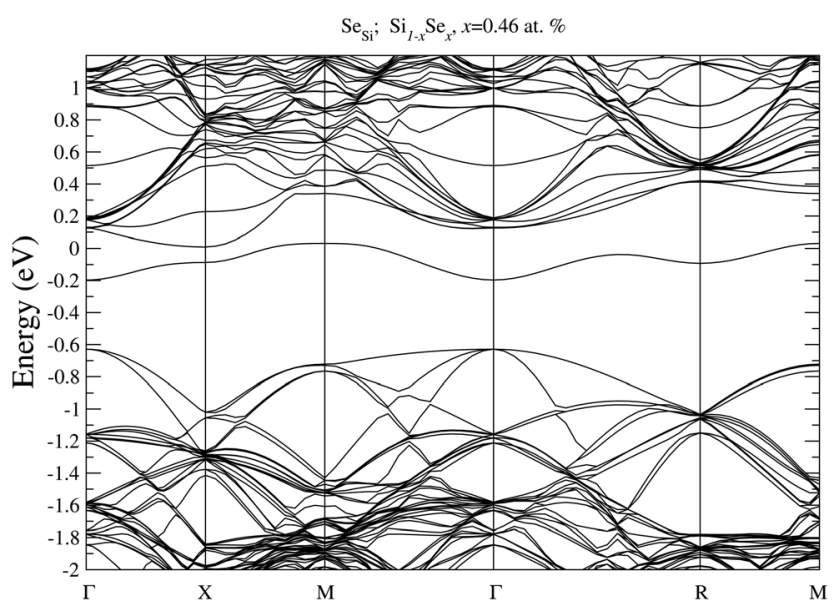

Fig. 5 Electronic bandstructure of Se hyperdoped $\mathrm{Si}_{1} \mathrm{Si}_{1-x} \mathrm{Se}_{x}$, for single $\mathrm{Se}_{\mathrm{Se}}$ at $x=0.46$ at $\%$. The zero of the energy scale corresponds to the Fermi energy.

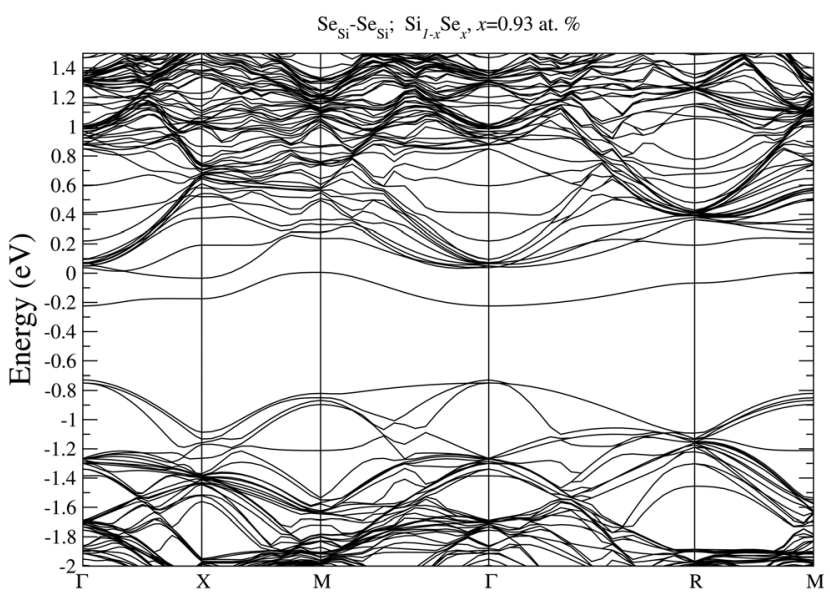

Fig. 6 Electronic bandstructure of Se hyperdoped $\mathrm{Si} \mathrm{Si}_{1-x} \mathrm{Se}_{x}$, for $\mathrm{Se}_{\mathrm{Se}^{-}}$ $\mathrm{Se}_{\text {se }}$ dimer at $x=0.93$ at $\%$. The zero of the energy scale corresponds to the Fermi energy.

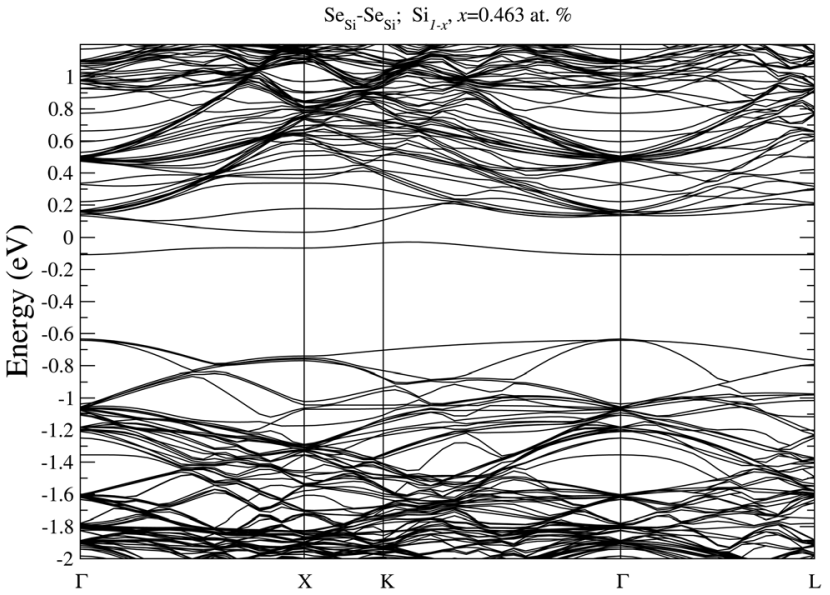

Fig. 7 Electronic bandstructure of $\mathrm{Se}$ hyperdoped $\mathrm{Si}, \mathrm{Si}_{1-x} \mathrm{Se}_{x}$, for substitutional $\mathrm{Se}_{\mathrm{Si}}-\mathrm{Se}_{\mathrm{Si}}$ dimer at $x=0.463$ at\%. The zero of the energy scale corresponds to the Fermi energy.

Fig. 6 we display the bandstructure of $\mathrm{Se}_{\mathrm{Si}}-\mathrm{Se}_{\mathrm{Si}}$ at $x=0.93$ at\%. The two bandstructures denote two systems very close to the
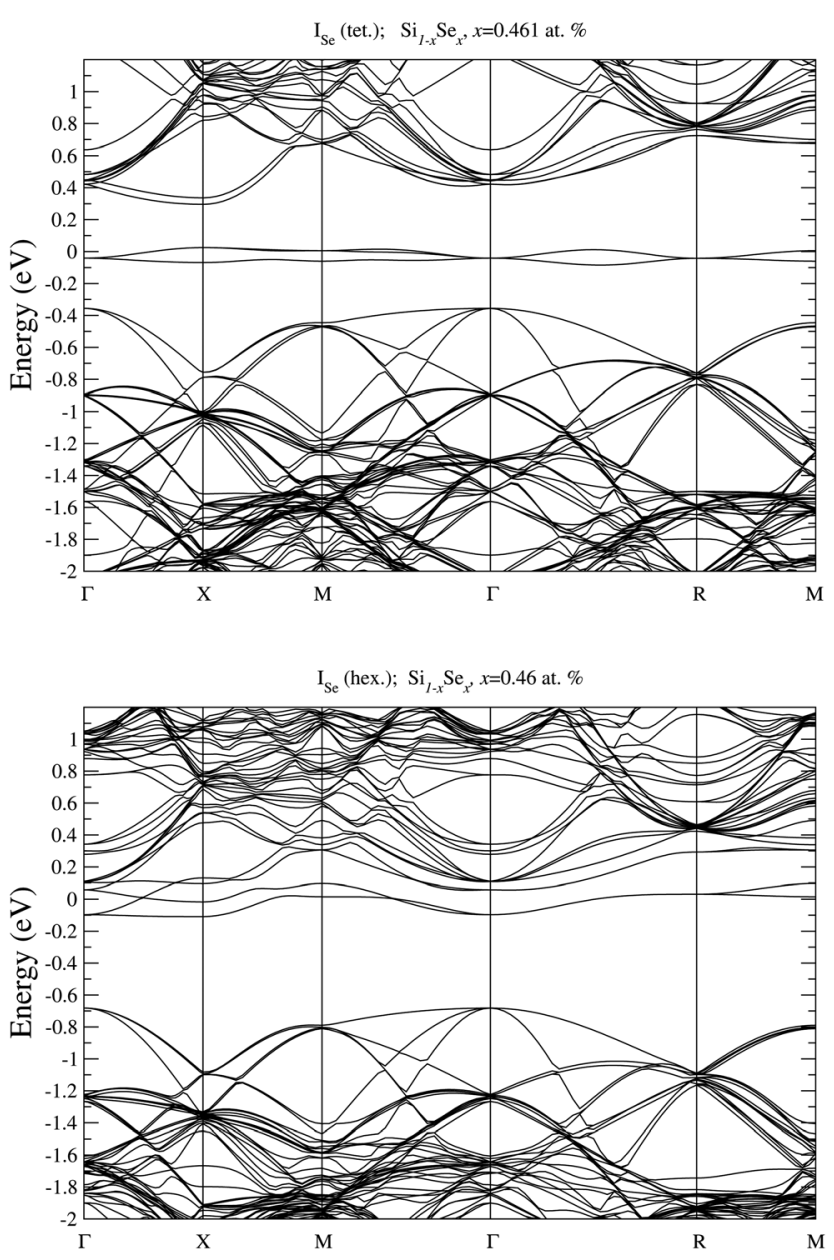

Fig. 8 Electronic bandstructure of Se hyperdoped $\mathrm{Si}_{1} \mathrm{Si}_{1-x} \mathrm{Se}_{x}$ for Se interstitial, $I_{\mathrm{Se}}$, in tetrahedral (top panel) and hexagonal position (bottom panel) at $x=$ $0.461 \mathrm{at} \%$. The zero of the energy scale corresponds to the Fermi energy. 

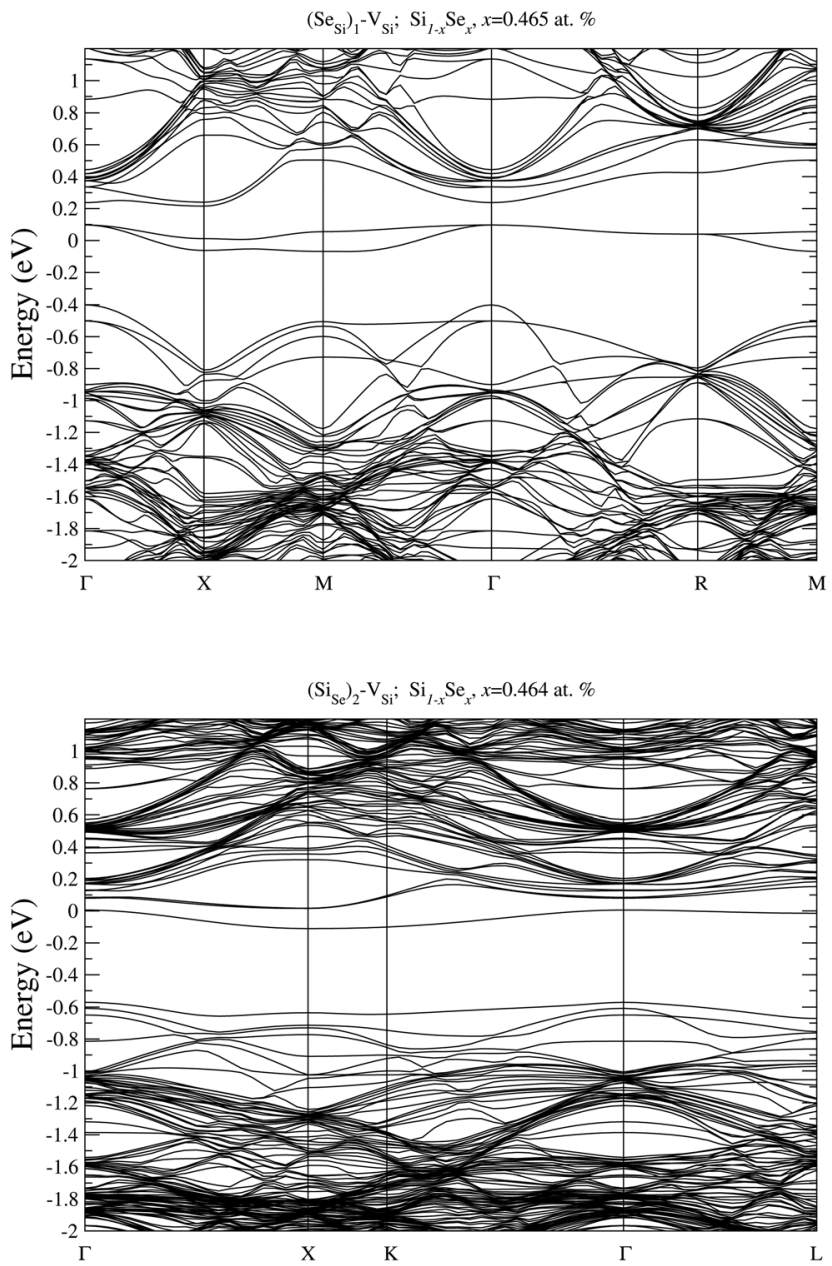

Fig. 9 Electronic bandstructure of Se hyperdoped $\mathrm{Si}, \mathrm{Si}_{1-x} \mathrm{Se}_{x}$, for $\left(\mathrm{Se}_{\mathrm{Si}}\right)_{1}-$ $V_{\mathrm{Si}}$ at $x \simeq 0.465$ at\% (top panel), and for $\left(\mathrm{Se}_{\mathrm{Si}}\right)_{2}-\mathrm{V}_{\mathrm{Si}}$ at $x \simeq 0.464$ at\% (bottom panel). The zero of the energy scale corresponds to the Fermi energy

semi-metallic state in which the conduction band minimum is basically equal to the maximum of the impurity band.

For completeness, by using the same Se concentration we computed the DOS displayed in Section III.B, for each type of defect we provide the electronic bandstructure, along high symmetry direction in the Brillouin zone (labels along high symmetry directions the Brillouin zone has been assigned according to the symmetry of the super-cell used in the simulation, see Appendix A for further details).

In Fig. 7 we display, the electronic bandstructure of $\mathrm{Se}_{\mathrm{Si}^{i}}-\mathrm{Se}_{\mathrm{Si}}$ for $x=0.463 \mathrm{at} \%$. At variance with bandstructure of the same complex for $x=0.93$ at $\%$ displayed in Fig. 6 , at the lower concentration of $x=0.463$ at $\%$, the IB is still separated by the conduction band.

In Fig. 8 we display the electronic bandstructure of $\mathrm{Se}$ interstitials. For $\mathrm{I}_{\mathrm{Se}}$ in the tetrahedral position we can notice the partially filled IB, which is situated deep in the bandgap, thus acting as an acceptor defect. At variance, at the considered concentration, the IB of $\mathrm{I}_{\mathrm{Se}}$ in hexagonal position, is merged into the conduction band, as can be noticed by looking
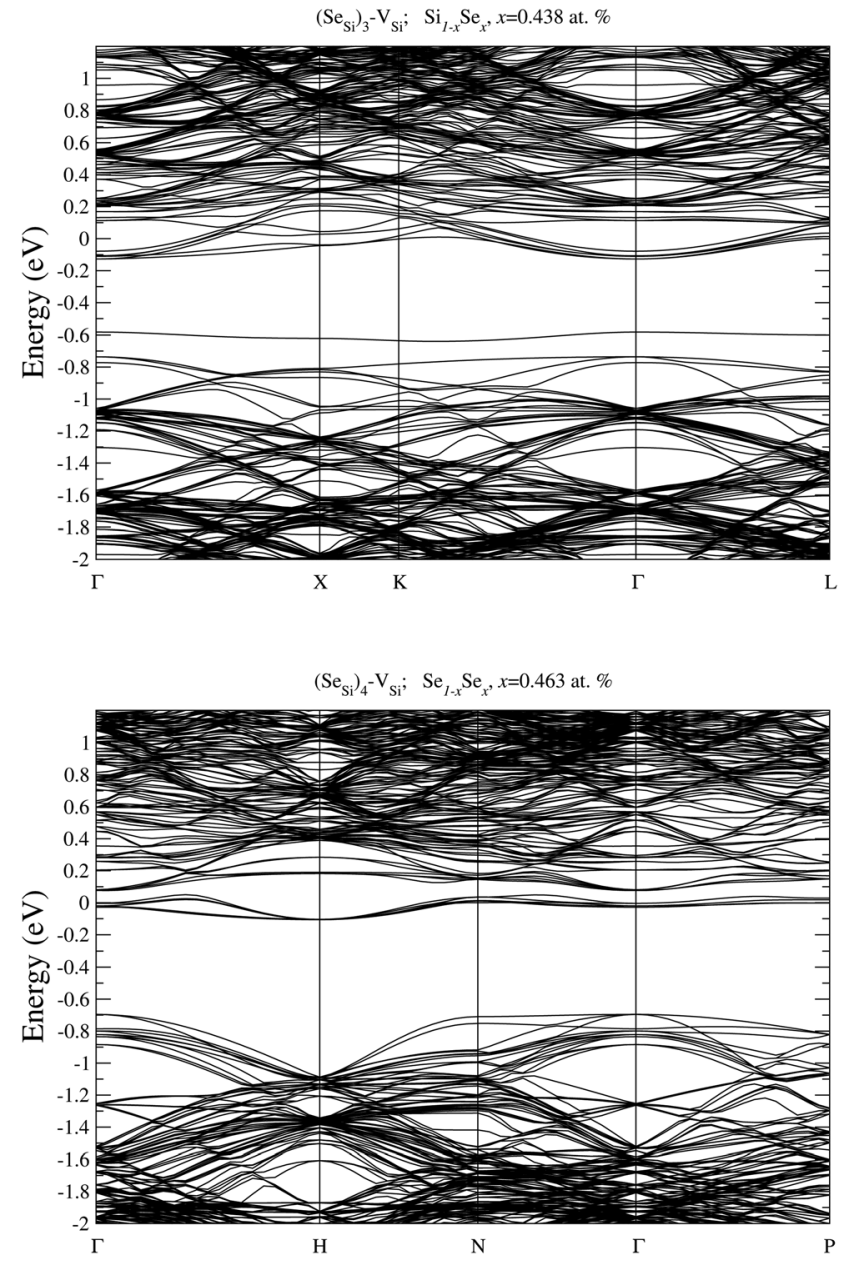

Fig. 10 Electronic bandstructure of Se hyperdoped $\mathrm{Si}, \mathrm{Si}_{1-x} \mathrm{Se}_{x}$, for $\left(\mathrm{Se}_{\mathrm{Si}}\right)_{3}-\mathrm{V}_{\mathrm{Si}}$ at $x \simeq 0.438$ at\% (top panel), and for $\left(\mathrm{Se}_{\mathrm{Si}}\right)_{4}-\mathrm{V}_{\mathrm{Si}}$ at $x \simeq 0.463$ at\% (bottom panel). The zero of the energy scale corresponds to the Fermi energy.

at the bandstructure along the $\Gamma-\mathrm{X}$ direction in the Brillouin zone.

In Fig. 9 and 10 we display the electronic bandstructure of the complexes $\left(\mathrm{Se}_{\mathrm{Si}}\right)_{m}-\mathrm{V}_{\mathrm{Si}}, m=1,2,3$, and 4 . As discussed in the main text, at $x \simeq 0.465$ at\%, the $\left(\mathrm{Se}_{\mathrm{Si}}\right)_{1}-\mathrm{V}_{\mathrm{Si}}$ is an acceptor complex, and it is characterized by a partially filled IB in the middle of the gap. At variance at $x \simeq 0.438$ at $\%$, the IB of $\left(\mathrm{Se}_{\mathrm{Si}}\right)_{3}-\mathrm{V}_{\mathrm{Si}}$ is fully merged into the conduction band. As can be noticed by looking at the figures, the considered concentrations $\left(\mathrm{Se}_{\mathrm{Si}}\right)_{2}-\mathrm{V}_{\mathrm{Si}}$ and $\left(\mathrm{Se}_{\mathrm{Si}}\right)_{4}-\mathrm{V}_{\mathrm{Si}}$ have shallow IBs, and these complexes act as donors. The bandstructures illustrated above provide a complementary picture of the analysis of electronic properties of the different types of defects discussed in Section III.B by means of the DOS.

\section{Appendix D: Formation energy of a couple of substitutional Se}

To support the assumption of a random distribution of Se dopants at the different lattice sites of silicon, used in Appendix $B$ to provide a quantitative estimation of the concentration of the different types of defects, we considered the formation 


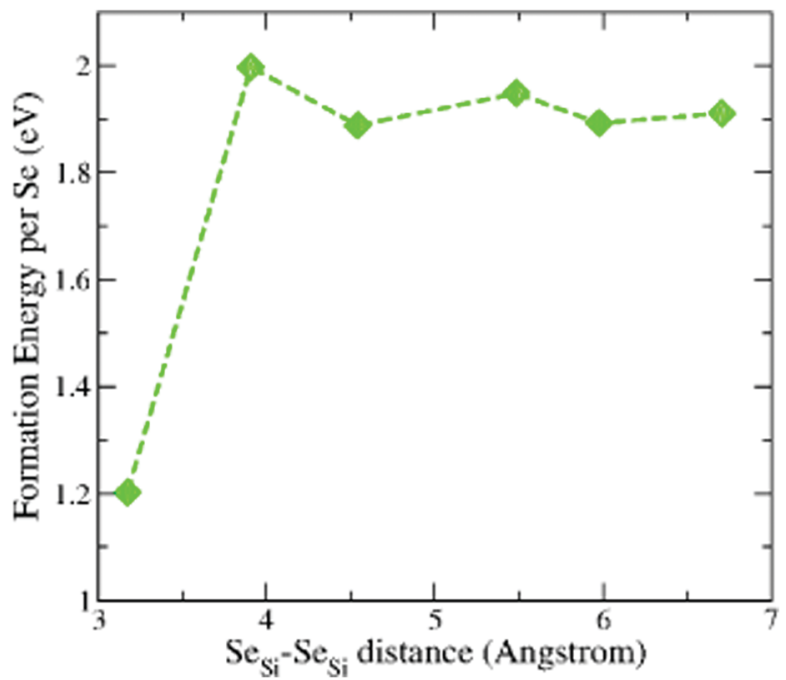

Fig. 11 Formation energy (per atom) of a couple of Se substitutional impurities in Se hyperdoped $\mathrm{Si}_{1} \mathrm{Si}_{1-x} \mathrm{Se}_{x} \mathrm{i}$, at $x=0.93$ at\%. One Se is placed at the $n$-neighbour shells $\left(\mathrm{NN}^{n}, n=1,2, \ldots, 6\right)$ surrounding the other Se. $\mathrm{Se}-\mathrm{Se}$ distance refers to relaxed positions and increases according to the increasing shell number $n$. Dashed line is a guide for the eye.

energy of a couple of $\mathrm{Se}_{\mathrm{Si}}$ placed at different distances at the substitutional sites of Si. The results for $x=0.93$ at $\%$ are displayed in Fig. 11. We considered the first Se placed at a substitutional site in the Si lattice site, while the second Se is placed at a substitutional site in one of the neighbouring shells of the Si lattice that surrounds the first Se. We considered an increasing number of next nearest neighbour shells $\mathrm{NN}^{n}, n=1$, $2, \ldots, 6$. We recall that $n=1$ corresponds to the $\mathrm{Se}_{\mathrm{Si}}-\mathrm{Se}_{\mathrm{Si}}$ (with the substitutional Se in a nearest neighbour shell). The lowest formation energy corresponds to the $\mathrm{Se}_{\mathrm{Si}}-\mathrm{Se}_{\mathrm{Si}}$ dimer, while all the other energies are comparable to the one of single $\mathrm{Se}_{\mathrm{Si}}$ at the same concentration (see Fig. 1).

From the figure we can conclude that while the dimer formation is energetically favored (probably due to the similar Se-Se bond length in the $\mathrm{Se}_{2}$ molecule with the $\mathrm{Si}-\mathrm{Si}$ bond length in bulk Si, see also Section 3.2) the positioning of Se at the other lattice sites can be considered, with a good approximation, equally probable, due to the very similar formation energies, thus justifying a distribution with equal probability of $\mathrm{Se}_{\mathrm{Si}}$ at this lattice site.

\section{Acknowledgements}

We acknowledge the CINECA for computer resources allocated under the ISCRA initiative (IMeCS project), and R. Colnaghi for technical support on computer hardware.

\section{References}

1 S. Hu, P. Han, S. Wang, X. Mao, X. Li and L. Gao, Improved photoresponse characteristics in se-doped si photodiodes fabricated using picosecond pulsed laser mixing, Semicond. Sci. Technol., 2012, 27, 102002.
2 Y. Berencén, S. Prucnal, F. Liu, I. Skorupa, R. Huebner, L. Rebohle, S. Zhou, H. Schneider, M. Helm and W. Skorupa, Room-temperature short-wavelength infrared si photodetector, Sci. Rep., 2017, 7, 43688.

3 M. Wang and Y. A. Berencén, Room-temperature infrared photoresponse from ion beam-hyperdoped silicon, Phys. Status Solidi A, 2021, 218, 2000260.

4 A. Luque, A. Marti and C. Stanley, Understanding intermediate-band solar cells, Nat. Photonics, 2012, 6, 146.

5 Y. Okada, N. J. Ekins-Daukes, T. Kita, R. Tamaki, M. Yoshida, A. Pusch, O. Hess, C. C. Phillips, D. J. Farrell, K. Yoshida, N. Ahsan, Y. Shoji, T. Sogabe and J. F. Guillemoles, Intermediate band solar cells: recent progress and future directions, Appl. Phys. Rev., 2015, 2(2), 021302.

6 M. Wang, A. Debernardi, Y. Berencén, R. Heller, C. Xu, Y. Yuan, Y. Xie, R. Bottger, L. Rebohle, W. Skorupa, M. Helm, S. Prucnal and S. Zhou, Breaking the doping limit in silicon by deep impurities, Phys. Rev. Appl., 2019, 11, 054039.

7 International Technology Roadmap for Semiconductors (ITRS), Tech. Rep. (Semiconductor Industry Association).

8 H.-J. Gossmann, C. S. Rafferty and P. Keys, Junctions for deep sub-100 nm mos: How far will ion implantation take us?, J. Mater. Res. Soc. Symp., 2000, 610, B1.2.1-B1.2.10.

9 H. Gossmann, F. C. Unterwald and H. S. Luftman, Doping of si thin films by lowtemperature molecular beam epitaxy, J. Appl. Phys., 1993, 73, 8237.

10 H. H. Radamson, M. R. Sardela Jr, L. Hultman and G. V. Hansson, Characterization of highly sbdoped si using highresolution xray diffraction and transmission electron microscopy, J. Appl. Phys., 1994, 76, 763.

11 K. Saarinen, J. Nissila, H. Kauppinen, M. Hakala, M. Puska, P. Hautojarvi and C. Corbel, Identification of vacancyimpurity complexes in highly n-type si, Phys. Rev. Lett., 1999, 82, 1883.

12 V. Ranki, K. Saarinen, J. Fage-Pedersen, J. Hansen and A. Larsen, Electrical deactivation by vacancy-impurity complexes in highly as-doped si, Phys. Rev. B: Condens. Matter Mater. Phys., 2003, 67, 041201.

13 M. Rummukainen, I. Makkonen, V. Ranki, M. J. Puska, K. Saarinen and H. J. L. Gossmann, Vacancy-impurity complexes in highly sb-doped si grown by molecular beam epitaxy, Phys. Rev. Lett., 2005, 94, 165501.

14 M. Ramamoorthy and S. Pantelides, Complex dynamical phenomena in heavily arsenic doped silicon, Phys. Rev. Lett., 1996, 76, 4753.

15 D. Mueller, E. Alonso and W. Fichtner, Arsenic deactivation in si: Electronic structure and charge states of vacancyimpurity clusters, Phys. Rev. B: Condens. Matter Mater. Phys., 2003, 68, 045208.

16 A. Satta, E. Albertazzi, G. Lulli and L. Colombo, Ab initio structures of asmv complexes and the simulation of rutherford backscattering channeling spectra in heavily as-doped crystalline silicon, Phys. Rev. B: Condens. Matter Mater. Phys., 2005, 72, 235206. 
17 D. Mathiot and J. C. Pfister, Diffusion of arsenic in silicon: validity of the percolation model, Appl. Phys. Lett., 1983, 42, 1043-1044.

18 D. Mathiot and J. C. Pfister, Dopant diffusion in silicon: a consistent view involving nonequilibrium defects, J. Appl. Phys., 1984, 55, 3518.

19 K. Pandey, A. Erbil, G. Cargill, R. Boehme and D. Vanderbilt, Annealing of heavily arsenic-doped silicon: Electrical deactivation and a new defect complex, Phys. Rev. Lett., 1988, 61, 1282-1285.

20 D. Lawther, U. Myler, P. Simpson, P. Rousseau, P. Griffin and J. Plummer, Vacancy generation resulting from electrical deactivation of arsenic, Appl. Phys. Lett., 1995, 67, 3575-3577.

21 V. Ranki, J. Nissila and K. Saarinen, Formation of vacancyimpurity complexes by kinetic processes in highly as-doped si, Phys. Rev. Lett., 2002, 88, 105506.

22 V. Ranki and K. Saarinen, Formation of thermal vacancies in highly as and p doped si, Phys. Rev. Lett., 2004, 93, 255502.

23 R. Pinacho, M. Jaraiz, P. Castrillo, I. Martin-Bragado, J. Rubio and J. Barbolla, Modeling arsenic deactivation through arsenic-vacancy clusters using an atomistic kinetic monte carlo approach, Appl. Phys. Lett., 2005, 86, 252103.

24 P. Voyles, D. Muller, J. Grazul, P. Citrin and H. Gossmann, Atomic-scale imaging of individual dopant atoms and clusters in highly n-type bulk si, Nature, 2002, 416, 826.

25 D. Mueller and W. Fichtner, Highly n-doped silicon: Deactivating defects of donors, Phys. Rev. B: Condens. Matter Mater. Phys., 2004, 70, 245207.

26 D. Chadi, P. Citrin, C. Park, D. Adler, M. Marcus and H. Gossmann, Fermi-level-pinning defects in highly ndoped silicon, Phys. Rev. Lett., 1997, 79, 4834.

27 M. T. Winkler, D. Recht, M.-J. Sher, A. J. Said, E. Mazur and M. J. Aziz, Insulator-to-metal transition in sulfur-doped silicon, Phys. Rev. Lett., 2011, 106, 178701.

28 E. Ertekin, M. T. Winkler, D. Recht, A. J. Said, M. J. Aziz, T. Buonassisi and J. C. Grossman, Insulator-to-metal transition in selenium-hyperdoped silicon: Observation and origin, Phys. Rev. Lett., 2012, 108, 026401.

29 M. Wang, A. Debernardi, W. Zhang, C. Xu, Y. Yuan, Y. Xie, Y. Berencén, S. Prucnal, M. Helm and S. Zhou, Critical behavior of the insulator-to-metal transition in Tehyperdoped si, Phys. Rev. B: Condens. Matter Mater. Phys., 2020, 102, 085204.

30 K. Suzuki, Y. Tada, Y. Kataoka, K. Kawamura, T. Nagayama, S. Nagayama, C. W. Magee, T. H. Bueyueklimanli, D. C. Mueller, W. Fichtner and C. Zechner, Maximum active concentration of ion-implanted phosphorus during solid-phase epitaxial recrystallization, IEEE Trans. Electron Devices, 2007, 54, 1985.
31 Z.-Y. Zhao and P.-Z. A. Yang, Insight into insulator-to-metal transition of sulfur-doped silicon by dft calculations, Phys. Chem. Chem. Phys., 2014, 16, 17499.

32 S. Zhou, F. Liu, S. Prucnal, K. Gao, M. Khalid, C. Baehtz, M. Posselt, W. Skorupa and M. K. Helm, Hyperdoping silicon with selenium: solid vs. liquid phase epitaxy, Sci. Rep., 2015, 5, 8329.

33 F. Liu, S. Prucnal, Y. Berencen, Z. Zhang, Y. Yuan, Y. Liu, R. Heller, R. Boettger, L. Rebohle, W. Skorupa, M. Helm and S. Zhou, Realizing the insulator-to-metal transition in sehyperdoped si via non-equilibrium material processing, J. Phys. D: Appl. Phys., 2017, 50, 415102.

34 See e.g., H. Overhof, M. Scheffler and C. M. Weinert, Formation energies, electronic structure, and hyperfine fields of chalcogen point defects and defect pairs in silicon, Phys. Rev. B: Condens. Matter Mater. Phys., 1991, 43, 12494, and references therein.

35 P. Giannozzi, S. Baroni, N. Bonini, M. Calandra, R. Car, C. Cavazzoni, D. Ceresoli, G. L. Chiarotti, M. Cococcioni, I. Dabo, A. D. Corso, S. Fabris, G. Fratesi, S. de Gironcoli, R. Gebauer, U. Gerstmann, C. Gougoussis, A. Kokalj, M. Lazzeri, L. Martin-Samos, N. Marzari, F. Mauri, R. Mazzarello, S. Paolini, A. Pasquarello, L. Paulatto, C. Sbraccia, S. Scandolo, G. Sclauzero, A. P. Seitsonen, A. Smogunov, P. Umari and R. M. Wentzcovitch, Quantum espresso: a modular and open-source software project for quantum simulations of materials, J. Phys.: Condens. Matter, 2009, 21, 395502.

36 A. M. Rappe, K. M. Rabe, E. Kaxiras and J. D. Joannopoulos, Optimized pseudopotentials, Phys. Rev. B: Condens. Matter Mater. Phys., 1990, 41, 1227(R).

37 D. Vanderbilt, Soft self-consistent pseudopotentials in a generalized eigenvalue formalism, Phys. Rev. B: Condens. Matter Mater. Phys., 1990, 41, 7892(R).

38 L. Kleinmann and D. Bylander, Efficacious form for model pseudopotentials, Phys. Rev. Lett., 1982, 48, 1425.

39 J. P. Perdew, K. Burke and M. Ernzerhof, Generalized gradient approximation made simple, Phys. Rev. Lett., 1996, 77, 3865; J. P. Perdew, K. Burke and M. Ernzerhof, Generalized gradient approximation made simple, Phys. Rev. Lett., 1997, 78, 1396(E).

40 R. W. Wyckoff, Crystal Structures, Wiley Interscience Publication, New York, 1971, vol. 1.

41 H. J. Monkhorst and J. D. Pack, Special points for brillouinzone integration, Phys. Rev. B: Condens. Matter Mater. Phys., 1976, 13, 5188.

42 G. Petretto, A. Debernardi and M. Fanciulli, Confinement effects and hyperfine structure in se doped silicon nanowires, Nano Lett., 2011, 11, 4509. 